\title{
Revealing Local Dynamics of Domain Growth in a Ferroelectric Material by In-situ STEM-SPM
}

\author{
H.J. Chang, ${ }_{*}^{*}$ S.V. Kalinin, ${ }^{*}$ P. Wu, ${ }^{* *}$ S.Y. Yang, ${ }^{* * *}$ P. Yu, ${ }^{* * *}$ R. Ramesh, ${ }^{* * *}$ L.Q. Chen, ${ }^{* *}$ S.J. \\ Pennycook ${ }^{*}$ and A. Borisevich ${ }^{*}$ \\ * Oak Ridge National Laboratory, Oak Ridge, TN 37831 \\ ** Pennsylvania State University, State College, PA 16802 \\ *** Department of Materials Science and Engineering and Department of Physics, University of \\ California, Berkeley, CA, 94720
}

The switchable polarization in ferroelectric (FE) materials enables their device applications such as FE random access memories and energy storage. Understanding the fundamental physics of ferroelectric domain stability and dynamics in the presence of electric fields is of crucial importance. Recently, piezoresponse force microscopy has emerged as a powerful tool for probing dynamics of bias-induced phase transitions in ferroelectrics. However, the limited spatial resolution $(10 \sim 30 \mathrm{~nm})$, and lack of structural information renders it virtually impossible to tie polarization dynamics to the specific structural elements. Here, we report direct observations of local structural changes through the thickness of a ferroelectric film induced by bias using in-situ scanning transmission electron microscopy (STEM). It enables visualization of domain growth along the film depth direction and the effect of slope of the existing domain walls for the new domain nucleation and its evolution in a micro ore even an atomic-scale.

We studied $300 \mathrm{~nm}$ thick multiferroic $\mathrm{BiFeO}_{3}$ (BFO) films which were epitaxially grown on [001] $\mathrm{DyScO}_{3}$ (DSO) substrate by chemical vapor deposition forming a system of $71^{\circ}$ domain walls. In-situ observation was carried out by using a Nanofactory Instruments Scanning Tunneling Microscope double tilt holder in a STEM (FEI Titan S 80-300) operated at $300 \mathrm{kV}$. Bias was applied through the $\mathrm{W}$ tip to the outer edge of the sample in the domain boundary region. The electric field is localized below the tip and hence polarization switching behavior is developed within a small volume.

Fig. 1(a) shows the domain structure of a BFO thin film before applying the bias. The angle between the domain walls and the film/substrate interface is $45^{\circ}$ or more. The tip end was placed at a domain wall. At $0.8 \mathrm{~V}$ a new domain nucleates and steadily increases in size under the applied bias. The domain growth is suppressed on the left side (Fig. 1(b)), due to a blocking effect from the neighboring domain wall. The original $71^{\circ}$ domain walls remain visible during the entire process, consistent with pinning by the surface. A much smaller domain wall pinning effect is observed as the new domain approaches the right hand ferroelectric domain wall (Fig. $1(\mathrm{c}))$, which is eventually penetrated, while the left side domain wall continues to block growth of the new domain (Fig. 1(d)). The experiments consistently show that the preferential nucleation site is at the acute angle between the domain wall and the surface, which is consistent with theoretical calculation that shows an asymmetric potential dip for nucleation near the twin domain boundary [1]. Hence, one direction is preferred for wall motion, resulting in asymmetric polarization dynamics. 
The observed switching behavior can be explained using the results of recent phase field modeling [2, 3]. An example of domain nucleation in the localized field of a scanning probe microscope tip is shown in Fig. 2. Switching results in a $71^{\circ}$ domain with narrow straight ferroelastic walls (Fig. 2(b)). Since the out-of-plane polarization component is not associated with long range strain fields, it would be invisible in a STEM image. However, the in-plane component of polarization would be visible in a STEM image. This component results in a nearsurface domain, with high-energy curved ferroelastic walls (Fig. 2(c)). This shape can be readily explained from consideration of electrostatic depolarization fields that result in the elongation along the (100) or (010) axis, i.e. in the direction of switched polarization component. To summarize, direct observation of in-situ nucleation and growth of ferroelastic domains unveils previously unknown domain switching mechanisms highly relevant to all multiaxial ferroelectric and multiferroic materials.

\section{References}

[1] S. Choudhury et al., Appl. Phys. Lett. 93 (2008) 3.

[2] P. Maksymovych et al., Science 324 (2009) 1421.

[3] N. Balke et al., Nat. Nanotechnol. 4 (2009) 868.

[4] This research is sponsored by the Division of Materials Sciences and Engineering, Office of BES of the U.S. DOE, and by appointment (H.J.C.) to the ORNL Postdoctoral Research Program administered jointly by ORNL and ORISE. Instrument access via ORNL's SHaRE User Facility, which is sponsored by the Scientific User Facilities Division, Office of BES, the U.S.DOE, is gratefully acknowledged.
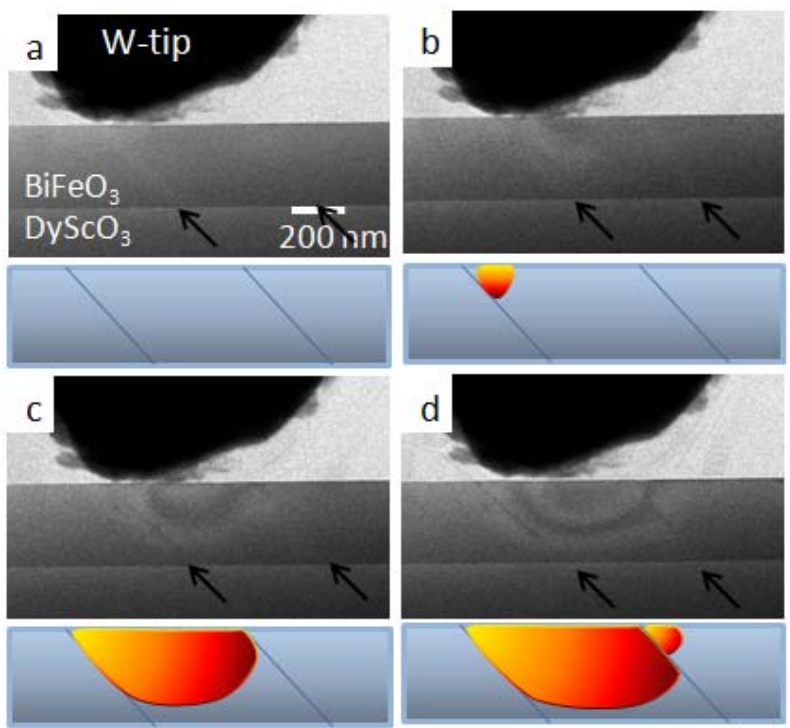

FIG. 1. (a) Bright field STEM image showing the $71^{\circ}$ domain wall structure of BFO film on DSO. (b-d) In situ imaging of bias induced domain nucleation and growth in a BFO thin film by STEM with corresponding schematics representing the domain nucleation and growth. The arrows indicate $71^{\circ}$ domain walls.
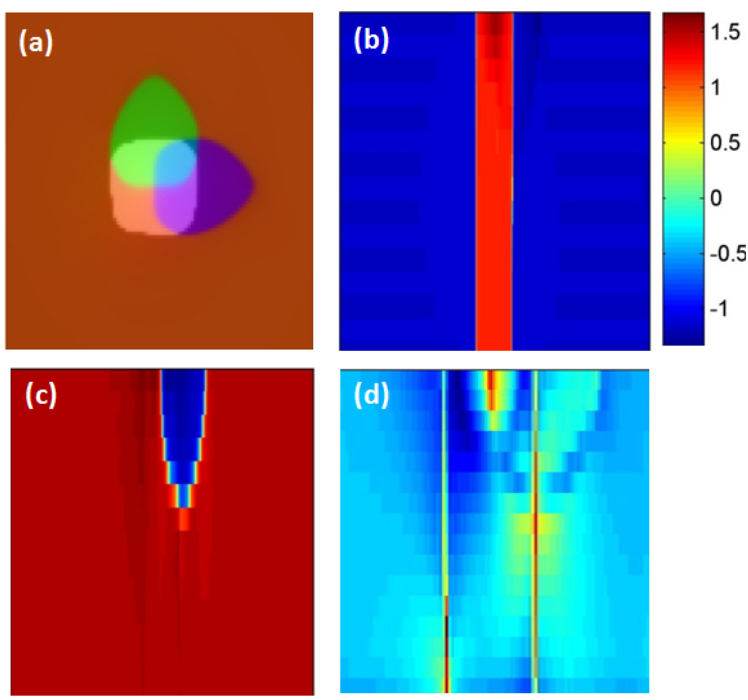

FIG. 2. Phase field modeling of the switching in BFO thin films. (a) Top view of the orthogonal domains: out-of-plane (pink) and two in-plane (green and blue) orientations. Side view of the BFO film showing (b) the out-of-plane component of polarization, which is normal to the tip; (c), in-plane component; and (d) elastic energy. 